Acta Crystallographica Section A

Foundations of

Crystallography

ISSN 0108-7673

Received 20 April 2005

Accepted 1 August 2005

(C) 2005 International Union of Crystallography Printed in Great Britain - all rights reserved

\section{Electron crystallography of zeolites - the MWW family as a test of direct 3D structure determination}

\author{
Douglas L. Dorset, ${ }^{\mathrm{a} *}$ Wieslaw J. Roth ${ }^{\mathrm{a}}$ and Christopher J. Gilmore ${ }^{\mathrm{b}}$

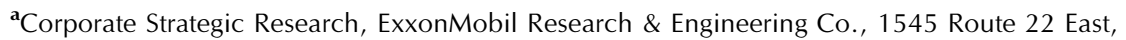 \\ Annandale, NJ 08801, USA, and ${ }^{\mathbf{b}}$ Chemistry Department, University of Glasgow, Glasgow \\ G12 8QQ, Scotland. Correspondence e-mail: d.I.dorset@exxonmobil.com
}

\begin{abstract}
The efficacy of direct methods for solving the crystal structures of zeolites from electron diffraction data is evaluated for a series of related materials, i.e. MCM22, MCM-49 and ITQ-1. First, it is established by tilting experiments that all materials share the same MWW framework. The calcined product of a delaminated MCM-22 precursor, ITQ-2, also shares this framework structure within the limited number of stacked unit cells. For all materials, the underlying space group is $P 6 / \mathrm{mmm}$ where $a \simeq 14.21, c \simeq 24.94 \AA$. Traditional direct methods are useful for determining the projected structure down the hexagonal axis but are not very effective for finding the three-dimensional structure. On the other hand, maximum-entropy and likelihood approaches are effective for determining either $2 \mathrm{D}$ projections or $3 \mathrm{D}$ frameworks. The major restriction to 3D determinations by direct methods is the limited goniometric tilt range of the electron microscope, hence the 'missing cone' of information. Potential maps from the most accurate phase sets are, therefore, observed as continuous density envelopes to the true structure. Some improvement is found when the Sayre equation predicts missing amplitudes and phases but it is clear that better specimen preparation methods are required to include projections containing the $c^{*}$ axis of the reciprocal lattice.
\end{abstract}

\section{Introduction}

Zeolites and other related microporous materials are extremely important in industrial processes since they provide the basis for materials separation and dimensionally constrained catalytic conversions (Szostak, 1998). Pore dimensionality, in addition to channel size, defines the potential utility of any new microporous framework, thus requiring crystallographic, as well as solid-state NMR, characterizations of tetrahedral site ( $\mathrm{Si}, \mathrm{Al}, \mathrm{P}$ etc.) connectivities (McCusker, 1991). Since zeolites are often (preferentially) microcrystalline, powder X-ray data are most often utilized for structure analysis and refinement. With several $a b$ initio techniques available for elucidating crystal structures from powder intensity data, the analyses are often quite routine. On the other hand, reflection overlap in powder patterns can sometimes frustrate the determination of unit-cell constants and space-group symmetry, as well as restrict the efficacy of a structure determination. Obviously, single-crystal intensity data would overcome these restrictions so that suitable crystals are often sought for X-ray data collection even if they are rather small (e.g. Morris et al., 2004).

Another possibility for single-crystal data collection is to exploit the enhanced scattering cross section of matter for electrons over X-rays (Vainshtein, 1964), so that single-crystal 3D electron diffraction intensities from readily available microcrystals would be employed for the structure determination. Certainly, as a qualitative tool, electron diffraction has often contributed dimensional and symmetry information for the indexing of powder X-ray patterns (Cheetham, 1995) but the actual quantitative use for structure analysis is less well developed. Typical crystal thicknesses for materials where $Z \simeq 14$ might be expected (Cowley \& Moodie, 1962) to lie adequately near the kinematical scattering limit for intermediate-voltage ( $\geq 200 \mathrm{kV}$ ) electron microscopes so that these intensities would be directly useful for structure analysis. By analogy, high-voltage electron microscopy of thin zeolite crystals has been quite effective for mapping out details of microporous networks (Thomas et al., 2001; Terasaki \& Ohsuna, 2003; Diaz et al., 2004).

The first application of electron diffraction intensities for determination of a zeolite structure via direct methods was made for MCM-22 (Nicolopoulos et al., 1995) in space group $P 6 / \mathrm{mmm}$. This was soon followed by the 3D direct analysis of SSZ-48 (Wagner et al., 1999) in space group $P 2_{1}$. Further analyses of various zonal projections, e.g. for MCM-22, MCM49 (Dorset, 2003d), ZSM-5, ZSM-11 (Dorset, 2003b) and mordenite (Dorset, 2003c), revealed that multiple-scattering effects other than coherent dynamical scattering, viz secondary scattering (Cowley et al., 1951), can degrade the observed selected-area diffraction intensities to the extent that only partial structure determinations might be possible. 
However, in optimal cases, such as the comparison of MCM-22 with MCM-49, it was thought that subtle structural differences might be justified by direct electron diffraction analysis (Dorset, 2003d) in a projection down the unit-cell hexagonal axis.

In this paper, the factors important for the realization of a meaningful 3D electron crystallographic analysis will be pursued further using the MWW family of zeolites, i.e. MCM22, MCM-49, ITQ-1, ITQ-2, as test materials. Interest in the assembly of layered precursors began with the synthesis of MCM-22 (Rubin \& Chu, 1990). The MCM-22 precursor comprises single aluminosilicate layers, postulated to be separated by an organic template and/or bonded by silanol groups (Roth \& Vartuli, 2002). Attempts have been made to characterize its structure (Njo et al., 1999). Upon calcination, free silanols are cross linked to form a three-dimensional framework, now designated as MWW (Baerlocher et al., 2001). Possible related materials, synthesized in other laboratories, include SSZ-25 (Zones et al., 1989), PSH-3 (Puppe \& Weisser, 1984) and ITQ-1 (Diaz-Cabanas et al., 2000), for example. If, instead of cross linking the layers, the assembly is delaminated, another material, MCM-56 (Fung et al., 1994) is produced after calcination; a related material, ITQ-2 (Corma et al., 1998, 2000) has been described. Insertion of pillars, after the layers have been separated by cationic surfactant molecules, produces another material, MCM-36 (Chu et al., 1994; Roth et al., 1995). Finally, it is also possible to synthesize the threedimensional MWW framework directly, yielding a material called MCM-49 (Bennett et al., 1993).

For the three-dimensional frameworks, the structural relationships between the variously synthesized materials has not been clearly established. A crystal structure has been reported (Leonowicz et al., 1994) for calcined MCM-22, for example. This model contains so-called 'detrital' atoms in addition to the aluminosilicate framework. The calcined pure silica analog, ITQ-1, has been shown (Camblor et al., 1998) to have the same hexagonal crystal structure as proposed for MCM-22 but without extra-framework atoms. The framework comprises intralayer sinusoidal 2D network channels with slightly elliptical 10-MR apertures. In addition there is a 'supercage' containing a 12-MR inner diameter. This becomes a 'cup' on the lamellar surface. The supercage does not communicate with the 2D intralayer channel network (Fig. 1).

The assignment of a space group for MCM-22, however, remains ambiguous (Leonowicz et al., 1994) since arguments have also been made to support a $C$-centered orthorhombic unit cell. High-resolution electron microscopy (Chan et al., 1995; Nicolopoulos et al., 1997) has found a similarity between ITQ-1 and SSZ-25 but could not establish their relationship to calcined MCM-22, for which high-resolution images have also been obtained to solve its crystal structure (Leonowicz et al., 1994). The possibility of a large pore along the [001] axis has been discussed (Chan et al., 1995; Nicolopoulos et al., 1997). (As shown below, this presumption is false.)

The relationship between calcined MCM-22 and calcined MCM-49 is not clear - do they have the same framework structure? Comparison of powder diffraction patterns presumably detected significant differences (Lawton et al., 1996) but an initial Rietveld refinement of the MCM-22 framework model against MCM-49 synchrotron powder data, on the other hand, established a close similarity. In an attempt to resolve differences with single-crystal electron diffraction intensities, there appeared to be a case for differentiating MCM-22 from MCM-49 (Dorset, 2003d), although the close relationship of MCM-22 to ITQ-1 seemed to be clear. However, the initial electron crystallographic study was based on data from untilted samples so that three-dimensional details could not be established.

In this paper, we address the following questions: can differences between the structures, suggested in earlier work, be firmly established; and is it possible to carry out a true $a b$ initio determination of a zeolite structure independent of powder X-ray data?

\section{Materials and methods}

\subsection{Zeolites}

Synthetic samples of MCM-22 and MCM-49, which had been prepared earlier at the former Mobil Research and Development Corporation, according to directions given in the relevant patents (Rubin \& Chu, 1990; Bennett et al., 1993), were used in their calcined forms. ITQ-1 was obtained from Dr Miguel Camblor (Instituto de Ciencia de Madrid, Madrid,

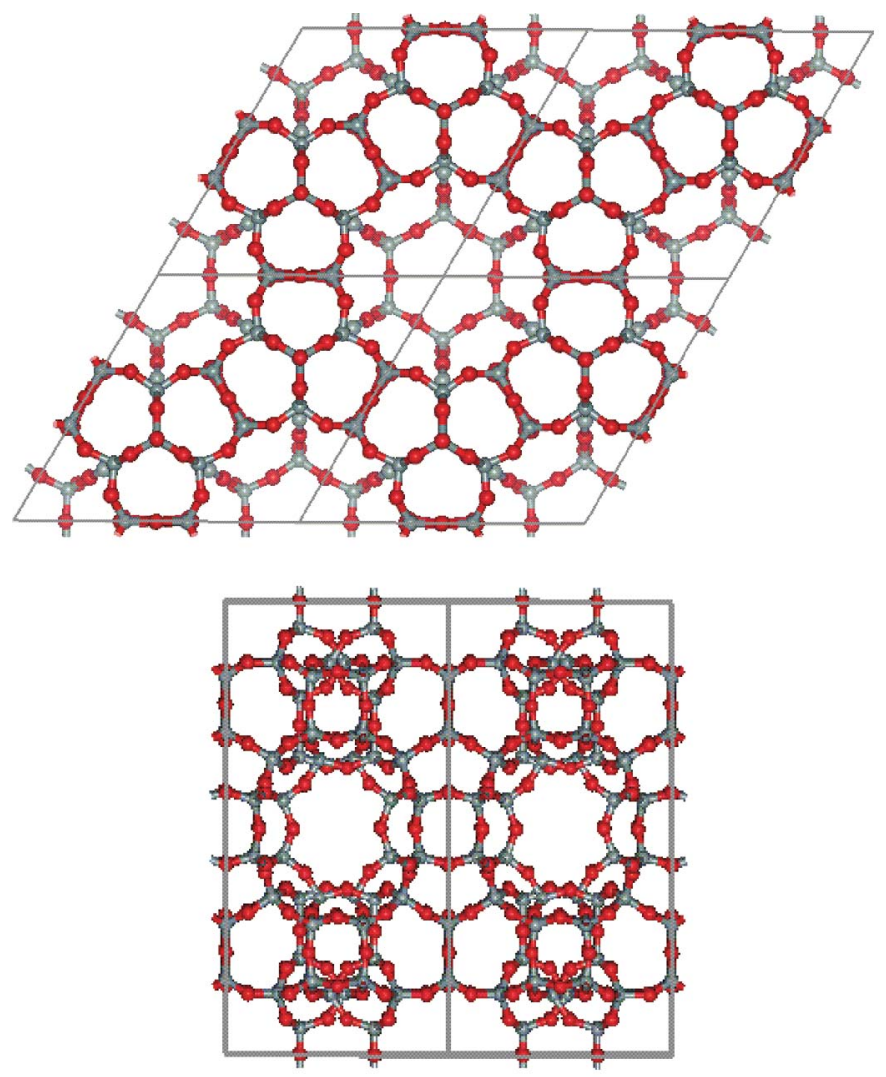

Figure 1

Framework model for MWW (ITQ-1). Top: [001] projection; bottom: projection down the $a$ axis. 
Spain) via Professor David Olson (Department of Chemical Engineering, University of Pennsylvania, USA) and ITQ-2 from Professor Avelino Corma (Instituto de Tecnologia Quimica, Valencia, Spain).

In typical preparations for electron diffraction, the zeolite was crushed with a mortar and pestle to a fine powder. The powder was then suspended in acetone by ultrasonication. A drop of the suspension was then allowed to dry on a carbonfilm-covered 300-mesh electron-microscope grid.

\subsection{Electron diffraction data collection}

Electron diffraction measurements were made originally at $200 \mathrm{kV}$ with a JEOL 2010 electron microscope $(\lambda=$ $0.02508 \AA$ ). Since this instrument was designed specifically for high-resolution electron microscopy, it included only a limited tilt capability for crystalline samples so that patterns were recorded only from untilted microcrystals. Data from this instrument were used in initial zonal structure determination/ refinement of MCM-22, MCM-49 and ITQ-1 (see below). This instrument has been recently replaced. Thus, using the goniometer stage of a $300 \mathrm{kV} \mathrm{FEI/Philips} \mathrm{CM-30} \mathrm{electron} \mathrm{micro-}$ scope $(\lambda=0.01969 \AA)$, successive electron diffraction patterns were recorded as individual microcrystals were tilted around the $a^{*}$ axis of the hexagonal unit cell. The tilt-axis limit to the goniometer is $\pm 60^{\circ}$. (This results in a $33 \%$ exclusion of the reciprocal lattice when sampling a platy crystal, thereby, in this case, excluding the $00 l$ reflections.) Patterns were recorded on Kodak SO-163 electron-microscopy film and subsequently developed in a Kodak HRP developer. Intensity data were extracted from the patterns after they were digitized on a flatbed transparency scanner by integration of diffraction spots with the program ELD in the CRISP package (Zou et al., 1993). Separate zonal patterns were scaled to one another by the sum of integrated axial reflections, $\sum I(h 00)$, facilitated also by the use of a fixed time to record the selected-area patterns at a constant illumination to the sample. No Lorentz correction was applied to the intensities, although there was a case for such a correction to the flat crystals from MCM-22 and MCM-49 based on the shape transform of a thin slab (Dorset, 2003d). With such a correction, the improvement to the intensity data is not significant for intermediate accelerating voltages since the deviation at higher diffraction angle can also be simulated by an overall temperature factor. On the other hand, there is no theoretical justification for the 'twobeam' assumption, $\mid F($ obs. $) \mid \sim k I$ (obs.), used in an earlier analysis of the MCM-22 structure (Nicolopoulos et al., 1995). This is appropriate for electron diffraction data from textures with large crystalline blocks (Vainshtein, 1964) but not for selected-area diffraction from single crystals.

As will be shown, the 3D intensity data from the three calcined zeolites (MCM-22, MCM-49, ITQ-1) are very similar to one another, partially because the electron scattering factors of $\mathrm{Al}$ and $\mathrm{Si}$ (Doyle \& Turner, 1968) are very similar. The fit of the combined observed structure factors to any framework model is made via $R(I)$ or $R(F)$, where $R(I, F)=\sum|| F_{h}^{\text {obs }}|-k| F_{h}^{\text {calc }}|| / \sum\left|F_{h}^{\text {obs }}\right|$ and $k$ is a scale factor to equalize the observed and calculated intensities or structure factors. As stated previously, the large values for these agreement factors arise from the perturbation of the intensity data by secondary scattering (Cowley et al., 1951). A closer fit of structure factors is found if only the most intense reflections are considered, since the relative effect of secondary scattering is most pronounced for the weaker reflections.

\subsection{Direct phase determination}

Zachariasen (1952) stated that a successful determination of any crystal structure requires finding accurate phase values for a small number ( $\mathrm{ca} 15 \%$ ) of reflections among the strongest normalized amplitudes. After that, the structure can be completed usually in a straightforward manner by successive approximations, e.g. by Fourier refinement. In 'traditional' probabilistic direct methods (Hauptman, 1972), often evaluated for zeolite electron diffraction data sets, one predicts the value of the phase invariant sum $\psi=\phi_{h}+\phi_{k}+\phi_{-h-k}$, where $h \cong h_{1} k_{1} l_{1}, k \cong h_{2} k_{2} l_{2}$. Prediction of $\psi$ (which is either 0 or $\pi$ in the centrosymmetric case) depends on the normalized magnitudes of reflections at these Miller indices, $\left|E_{h}\right|^{2}=\left|F_{h}^{\mathrm{obs}}\right|^{2} / \varepsilon \sum_{i} f_{i}^{2}$, and the associated concentration variable, $A=\left(2 / N^{1 / 2}\right)\left|E_{h} E_{k} E_{h+k}\right|$. In the preceding, $f_{i}$ are electron scattering factors (Doyle \& Turner, 1968) and $N$ is the number of atoms in the unit cell. Starting information for the phasing process can utilize a small number of phase values assigned to reflections with appropriate index parity to define the unit-cell origin (Hauptman, 1972); similarly, a single reflection phase in some non-centrosymmetric structures can be used to define the enantiomorph. Algebraic direct methods, such as the Sayre (1952) equation, behave in a similar fashion, since the invariant relationships are generated by the convolution $F_{h}=(\theta / V) \sum_{k} F_{k} F_{h-k}$.

The success of this phasing approach in electron crystallography (Dorset, 1995), especially in the guise of symbolic addition (Karle \& Karle, 1966) or with the tangent formula (Karle \& Hauptman, 1956) or the Sayre (1952) equation, is based on the observation that strong structure-factor magnitudes often remain strong even after multiple-scattering perturbations. [Similar observations have been made by other researchers studying inorganic structures with electron diffraction intensities (e.g. Weirich, 2001).] This is equivalent to saying that the experimental Patterson function still contains most of the information to be found in the crystal autocorrelation function (Dorset, 2003a). Strict adherence to the kinematical scattering limit is not required and a few erroneous phase estimates can be tolerated although, in some cases, multiple scattering can cause the appearance of spuriously strong reflections to lead the phase determination astray (Dorset, 2003c). Other causes for failed determinations include incomplete data sets leading to poor vector connectivity via Miller indices.

Another way of approaching the phase problem that is not restricted by vector connectivity of reflections in the data set (i.e. to construct invariant sums) is to simply carry out a set of phase permutations and combinations for an identified 
number $n$ of strong reflections. This simple device was used effectively by Robertson \& White (1945) in the analysis of the coronene structure well before direct methods were invented. In the approach employing maximum entropy and likelihood to evaluate the phasing trials (Bricogne \& Gilmore, 1990; Gilmore et al., 1990), the computer program MICE (Maximum entropy In a Crystallographic Environment) (Gilmore \& Bricogne, 1997) was employed. It is first assumed that there are a small number of reflections in a basis set $\{H\}$ whose phases are known a priori. (These can include origin-defining phases as before.) The task then is to find the phases of reflections in the unknown set $\{K\}$ from the known set $\{H\}$. We define the unitary structure factor in $P 1, \quad\left|U_{h}\right|^{\text {obs }}=$ $\left|E_{h}\right|^{\text {obs }} / N^{1 / 2}$, then the task is to extrapolate the known information into the unknown set. The FT of the map should also yield a close fit of $\left|U_{h}\right|^{\text {obs }}$ to $\left|U_{h}^{\mathrm{ME}}\right|$ and the entropy maximization evaluates this via a reduced $\chi^{2}$ statistic, whose optimal value should be unity provided that viable errors can be established for the unitary structure factors. If the number of starting reflections is small, the phasing power of a maximumentropy map $q^{\mathrm{ME}}(x)$ is rather weak when its Fourier transform (FT) is calculated. Entropy maximization of the map involves an optimization step that naturally enforces map positivity, as is discussed by Bricogne \& Gilmore (1990). It is therefore beneficial to insert $n$ algebraic phase terms into the basis set for other strong reflections in the data set. If there are $n(c)$ centrosymmetric phases (whose values are restricted to $0, \pi$ ) and $n(a)$ non-centrosymmetric phases (whose values are estimated by quadrant permutations: $\pm \pi / 4, \pm 3 \pi / 4)$ to be inserted into the basis set, we then generate $2^{n(c)}, 4^{n(a)}$ possible phase combinations and obtain multiple basis sets, each one of which needs to be subjected to entropy maximization.

The entropy optimization subjects the map entropy, $S=-\sum_{i} p_{i} \ln p_{i}$ (where $p_{i}$ are normalized map pixel values), to a constrained maximization in which constraints are the unitary structure factors of the relevant basis set. It is necessary to select the optimum phase solution from the multiple phase sets. Map density flatness has often been cited as a suitable FOM to recognize viable phase combinations (Luzzati et al., 1988; Sato, 1992), a likelihood measure is preferred for this identification (Gilmore et al., 1990; Sayre, 1993). One can define the likelihood for the centrosymmetric case (Gilmore, 1998):

$$
\begin{aligned}
\Lambda_{K}= & \frac{2\left|U_{k}\right|^{\mathrm{obs}}}{\pi\left(2 \varepsilon_{K} \Sigma+\sigma_{K}^{2}\right)} \exp \left\{-\frac{1}{2} \frac{\left(\left|U_{K}\right|^{\text {obs }}\right)^{2}+\left(\left|U_{K}^{\mathrm{ME}}\right|\right)^{2}}{2 \varepsilon_{K} \Sigma+\sigma_{K}^{2}}\right\} \\
& \times \cosh \left(\frac{\left|U_{K}\right|^{\mathrm{obs}}\left|U_{K}^{\mathrm{ME}}\right|}{2 \varepsilon_{K} \Sigma+\sigma_{K}^{2}}\right) .
\end{aligned}
$$

This is compared to the null estimate $\Lambda_{K}^{0}$, evaluated for $\left|U_{K}^{\mathrm{ME}}\right|=0$ with the quantity $L_{K}=\log \left[\Lambda_{K} / \Lambda_{K}^{0}\right]$ via the global $\log$-likelihood gain LLG $=\sum_{K} L_{K}$. (The subscript $K$ refers to reflections in the non-basis set.) Sometimes a FOM combining the LLG and the entropy value $S$ is evaluated as $N S+$ LLG, where $N$ is the number of atoms in the unit cell. MICE treats this number as a refinable variable, starting with the actual $N$ when optimizing the likelihood (Gilmore, 1998).

As is apparent, a large number of trial phases $[n(a)+n(c)]$ can, by permutation and combination, lead to a very large number of phase sets, even if the structure is centrosymmetric. For this reason, various error-correcting codes are employed (Gilmore et al., 1999) to reduce the number of trials, accepting that a very small number of phases might be in error. For example, a Hadamard-Hamming code can be applied, in which only 16 phase trials are evaluated instead of $2^{8} \cong 256$ permutations for 8 reflections. [This use of coding theory in this context had been discovered independently by Woolfson (1954).] The best phase set has two or fewer errors. Similarly, a Nordström-Robinson (1967) code can be explored, permitting 16 phase terms to be evaluated. Instead of exploring $2^{16} \cong$ 65536 permutations, only 256 trials need be considered with a maximum of four errors in the best solution. Finally, a Golay (1949) code will generate 4096 phase combinations for 24 reflections instead of $2^{24} \cong 16777216$ trials with a maximum number of four incorrect assignments. In this paper, phase errors are reported as unweighted r.m.s. values, compared to $90^{\circ}$ for a random determination.

\section{Results}

We now present structure solutions for ITQ-1, MCM-49, MCM-22, ITQ-2 and a composite MWW data set, evaluating symbolic addition, the Sayre equation and maximum-entropy methods.

\subsection{ITQ-1}

The crystal structure of ITQ-1 (Fig. 1) has been reported (Camblor et al., 1998) based on a Rietveld refinement of synchrotron powder diffraction data, starting with the MCM22 framework without 'detrital' atoms. The reported cell constants in space group P6/mmm are: $a=14.21, c=24.94 \AA$. Electron diffraction tilts of single microcrystals in the electron microscope give values: $a=14.48 \pm 0.12, c=26.0 \pm 0.6 \AA$; the corresponding unique $3 \mathrm{D}$ data set comprises 120 unique reflections with intensities conforming to the $P 6 / \mathrm{mmm}$ symmetry rules. It is clear from evaluation of tilted projections that there is also a mirror relationship of intensity values at

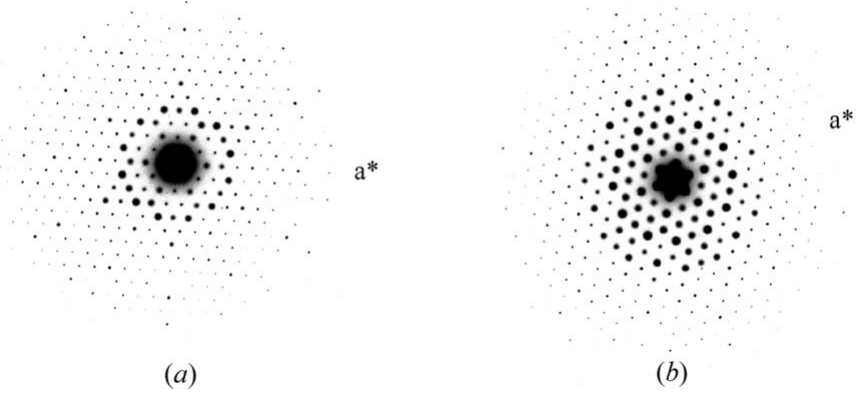

Figure 2

Electron diffraction patterns ( $h k 0)$ from ITQ-1. (a) Thin crystals; $(b)$ thick crystals. 
equal \pm tilt values around $a^{*}$ from the $h k 0$ reciprocal-lattice net. Aside from the $h k 0$ data (Fig. $2 a$ ), representative patterns from tilted projections (Fig. 3) include, for this zeolite, the $h k k$, $h, k, 2 k, h, 3 n, 2 n, h, 2 n, n$ and $h, 3 n, n$, within a maximum tilt of $55.5^{\circ}$. (As reference points, the $h, k, 2 k$ pattern is found at $48.7^{\circ}$ tilt, whereas the $h k k$ pattern is found at $29.7^{\circ}$ tilt around the $a^{*}$ axis.) Thus, structure-factor amplitudes conform to the relationships: $|F(h, k, l)|=|F(-h,-k,-l)|=|F(h, k,-l)|=|F(k, i, l)|=$ $|F(k, h, l)| \neq|F(-h, k, l)| ;|F(-h, k, l)|=|F(h,-k, l)|$, and are consistent with space group P6/mmm (No. 191) (Hahn, 1995).

Initially, a zonal structure was determined by probabilistic direct methods using $200 \mathrm{kV}$ data. In general, some of the microcrystals used for electron diffraction studies were somewhat thicker than those employed in an earlier investigation of MCM-22 and MCM-49 (Dorset, 2003d). (This was estimated qualitatively from the transparency of the thinnest crystalline samples to the electron beam.) Two kinds of hexagonal electron diffraction pattern were obtained from ITQ-1, both corresponding to $p 6 \mathrm{~mm}$ projected symmetry (Fig. 2 ) and both are consistent with the originally reported cell constant $a=14.21 \AA$. In both, the diffraction resolution was somewhat higher than observed (Dorset, 2003d) for the other MWW zeolites $\left(d_{\min }=0.79,1.23\right.$ and $1.54 \AA$, respectively, for ITQ-1, MCM-22 and MCM-49).

For the pattern represented by Fig. 2(a), the overall temperature factor derived from a Wilson (1942) plot was $B$ $0.0 \AA^{2}$. In earlier work on organics (Dorset, 1995), this unusually low temperature factor often indicated that some form of multiple-scattering perturbation to the intensities was present. A structure-factor calculation with the atomic coordinates of the ITQ-1 model in Table 1 (Camblor et al., 1998), using $B_{\text {iso }}=2.0 \AA^{2}$ for both atom types, shows that the agreement $[R(F)=0.27]$ is nearly as good as found earlier for the MCM-22 and MCM-49 examples (Dorset, 2003d) and certainly better than found with electron diffraction data from many other zeolites (Dorset, 2003b,c). Improved fit of calculated and observed amplitudes [e.g. $R(F)=0.16]$ was found after a correction for secondary scattering (Cowley et al.,
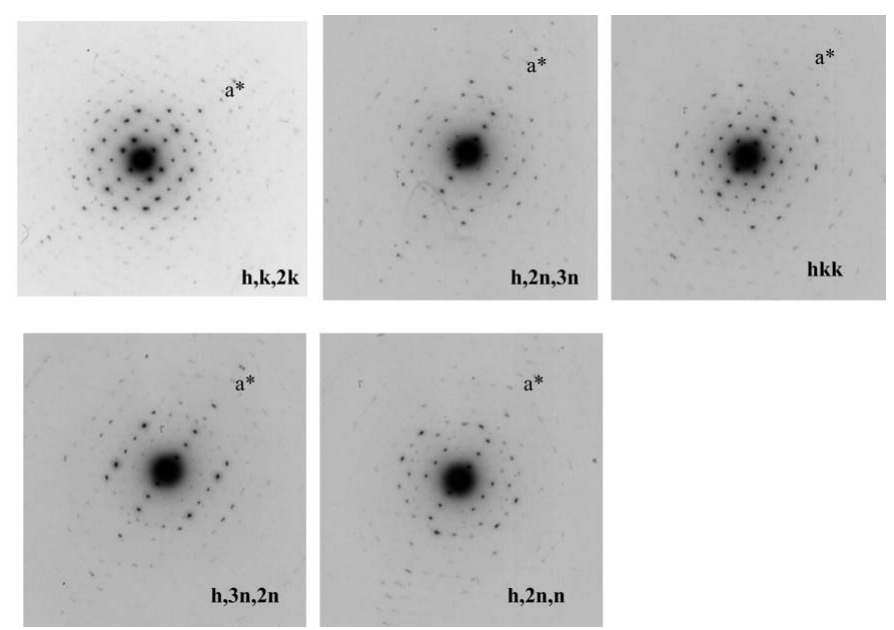

Figure 3

Tilted projections of MWW reciprocal-lattice around $a^{*}$.
Table 1

Structure factors for composite MWW (correct phases, bold indicates where false Fourier refinement values occur).

\begin{tabular}{|c|c|c|c|c|c|c|c|}
\hline$h k l$ & Fobs & $h k l$ & Fobs & $h k l$ & Fobs & $h k l$ & Fobs \\
\hline 100 & -25.38 & 611 & -6.97 & 042 & 3.14 & 114 & -8.54 \\
\hline 200 & -15.49 & 711 & -3.99 & 432 & 5.37 & 024 & 13.16 \\
\hline 300 & 15.75 & 811 & -3.14 & 532 & -6.55 & 124 & -16.29 \\
\hline 400 & -6.49 & 911 & -1.92 & 632 & -2.59 & 224 & 4.45 \\
\hline 500 & 11.97 & 021 & 11.28 & 732 & -2.48 & 324 & 5.45 \\
\hline 600 & 3.85 & 221 & 10.83 & 033 & 6.25 & 424 & -4.26 \\
\hline 700 & 6.78 & 321 & -5.81 & 133 & 4.88 & 524 & $\mathbf{3 . 3 7}$ \\
\hline 800 & 10.34 & 421 & -3.89 & 333 & 2.87 & 624 & -3.88 \\
\hline 900 & -4.51 & 521 & 7.71 & 433 & 6.23 & 724 & -2.69 \\
\hline 110 & -8.03 & 621 & 2.99 & 533 & -3.99 & 824 & 2.89 \\
\hline 210 & 8.29 & 721 & -2.84 & 633 & 3.10 & 164 & -3.24 \\
\hline 310 & -22.23 & 821 & 4.67 & 733 & -4.43 & 064 & 8.32 \\
\hline 410 & 5.61 & 031 & 18.98 & 043 & -7.07 & 154 & -5.91 \\
\hline 510 & 4.78 & 431 & -5.17 & 143 & 7.34 & 235 & 4.09 \\
\hline 610 & -8.44 & 531 & 6.23 & 443 & 1.98 & 145 & -2.87 \\
\hline 710 & 5.99 & 012 & 15.65 & 543 & -1.68 & 055 & 4.52 \\
\hline 810 & -7.06 & 112 & 12.03 & 113 & 7.57 & 155 & 3.98 \\
\hline 220 & 15.31 & 212 & -12.03 & 023 & -10.25 & 255 & $-\mathbf{3 . 9 7}$ \\
\hline 320 & 10.65 & 312 & 13.49 & 123 & 6.77 & 355 & 2.36 \\
\hline 420 & -6.82 & 412 & 5.18 & 223 & 11.66 & 455 & -4.53 \\
\hline 530 & 7.54 & 512 & 3.80 & 323 & -13.70 & 126 & 9.64 \\
\hline 620 & 4.18 & 612 & 7.15 & 423 & 2.66 & 036 & 6.33 \\
\hline 720 & -6.09 & 712 & -3.86 & 044 & -13.91 & 136 & -8.07 \\
\hline 330 & 14.80 & 812 & 4.70 & 144 & 3.99 & 236 & -4.32 \\
\hline 430 & -9.29 & 022 & -15.97 & 444 & -5.33 & 336 & 2.88 \\
\hline 530 & -5.77 & 222 & 8.33 & 544 & -3.29 & 436 & -4.39 \\
\hline 630 & -4.75 & 322 & 4.65 & 644 & 3.30 & 226 & 3.67 \\
\hline 440 & 8.92 & 422 & 4.97 & 034 & 12.67 & 046 & 7.03 \\
\hline 011 & -12.37 & 522 & -7.66 & 134 & 10.47 & 146 & -2.35 \\
\hline 111 & 11.90 & 622 & -5.52 & 334 & -5.18 & 246 & 3.48 \\
\hline 211 & 8.17 & 722 & 4.09 & 434 & -2.92 & 536 & -1.51 \\
\hline 311 & -10.92 & 822 & 4.49 & 534 & 6.37 & 156 & -3.00 \\
\hline 411 & 3.11 & 032 & -21.92 & 634 & 2.69 & 066 & -7.52 \\
\hline 511 & 2.87 & 332 & -6.18 & 734 & -2.84 & 446 & 3.31 \\
\hline 166 & -2.45 & 077 & 2.56 & 148 & -3.98 & 249 & 6.10 \\
\hline 266 & 3.94 & 177 & -3.68 & 248 & -3.49 & 159 & -2.90 \\
\hline 347 & -7.53 & 228 & -8.61 & 348 & 2.69 & 069 & -4.23 \\
\hline 257 & 3.00 & 138 & 3.97 & 448 & 2.99 & 169 & 3.14 \\
\hline 167 & 2.69 & 048 & 6.38 & 339 & 8.37 & & \\
\hline
\end{tabular}

1951). A number of patterns could be found that resemble Fig. $2(a)$ and the intensities were consistent with those found for this pattern [e.g. $R(F)=0.11]$.

A direct determination of crystallographic phases was performed by symbolic addition (Karle \& Karle, 1966); there were only two $\Sigma_{2}$ triple estimates within the 34 with largest $A$ value that disagree with the previously obtained phase model. Initially, two algebraic unknowns were employed to evaluate new phase values by symbolic addition but the value of one of these was found during the course of the evaluation. There are no allowable origin-defining phases for $h k 0$ data in this space group (Rogers, 1980). The initial phasing model comprised 26 reflections of the total 61 , only one of which had a phase value disagreeing with the initial structural model (Camblor et al., 1998). Fourier refinement led to the map in Fig. 4 wherein five unique Si-atom sites were located as well as three O-atom positions. In general, the ITQ-1 cage model satisfies the $h k 0$ intensities. The mean deviation for $T$-atom sites not on special positions is $0.40 \AA$; for $\mathrm{O}$ atoms this is $0.11 \AA$. The agreement of the derived coordinates with the experimental intensity data, using $B_{\text {iso }}=2.0 \AA^{2}$, is $R=0.39$. Obviously, the 
complete structural details are not recovered from Fourier refinement.

The second diffraction pattern (Fig. $2 b$ ) could also be reproduced with somewhat less self-consistency $[R(F) \leq 0.19]$ than the one in Fig. 2(a). The fit of the ITQ-1 model in a structure-factor calculation is less favorable than before $[R(F)=0.55]$. Nevertheless, a Wilson (1942) plot finds a physically more 'reasonable' value for the overall temperature factor $\left(B=2.9 \AA^{2}\right)$. Direct phase determination reveals that, within the $31 \Sigma_{2}$ triples with largest $A$ values there are four that disagree with the ITQ-1 phases calculated from the structural model. The derived phasing model of 21 reflections contains four phase terms that disagree with the original ITQ1 model. $T$-atom sites in the potential map are reasonably close to the ideal positions, but the peaks are distorted. Also there are other peak positions in the map that would not correspond to any reasonable linkage to O-atom sites.

In an attempt to determine the 3D structure by maximumentropy and likelihood methods via the Nordström-Robinson code for 16 phase generators were used, the resultant phase errors were $\langle\Delta \phi\rangle=48.0$ and $80.9^{\circ}$, respectively, for $h k 0$ and $h k l$ data, the 3D set containing 69 phased amplitudes. For the $\mathrm{X}$-ray structure model, the fit to the 3D electron diffraction amplitudes is $R(I)=0.35, R(F)=0.38$, or, if the subset of 36 reflections where $|F \mathrm{obs}| \geq 10$ is monitored, $R(I)=0.26, R(F)=$ 0.27 . Better results were obtained from a larger MCM-49 3D data set (see below).

\subsection{MCM-49}

Given a characteristic hexagonal $h k 0$ electron diffraction pattern (similar to Fig. $2 a$ ) with average axial value $a=$ $14.07 \pm 0.16 \AA$, the zonal projection structure had been determined by conventional direct methods (Dorset, 2003d). The symmetry of the zonal pattern is obviously $p 6 \mathrm{~mm}$. Tilting experiments on individual microcrystals around the $h 00$

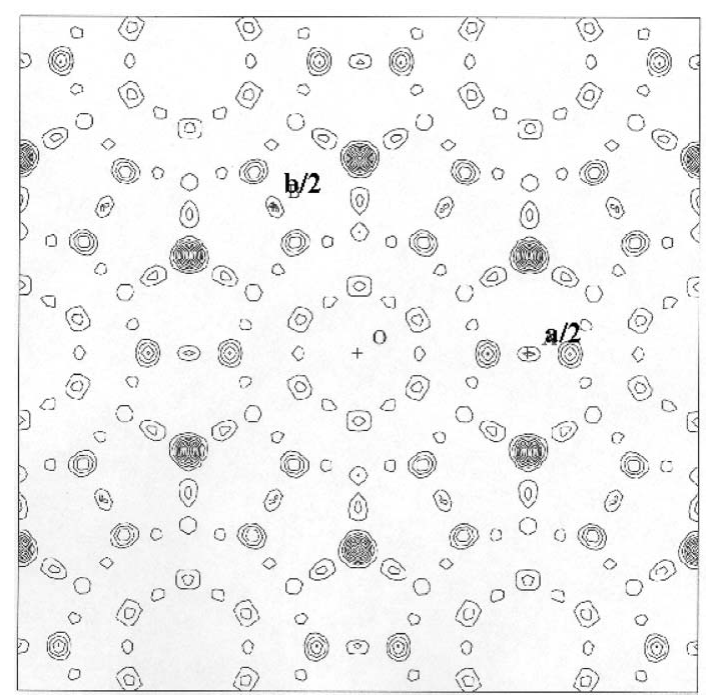

Figure 4

Projected potential map for ITQ-1 after direct determination by symbolic addition.
Table 2

Atomic coordinates for MWW materials.

\begin{tabular}{|c|c|c|c|c|c|c|}
\hline & \multicolumn{3}{|c|}{$\begin{array}{l}\text { ITQ-1 } \\
\text { (Camblor } \text { et al., 1998) }\end{array}$} & \multicolumn{3}{|c|}{$\begin{array}{l}\text { Composite MWW } \\
\text { (electron diffraction study) }\end{array}$} \\
\hline & $x / a$ & $y / b$ & $z / c$ & $x / a$ & $y / b$ & $z / c$ \\
\hline Si1 & 0.6667 & 0.3333 & 0.0633 & 0.667 & 0.333 & 0.065 \\
\hline $\mathrm{Si} 2$ & 0.4685 & 0.2342 & 0.1356 & 0.519 & 0.260 & 0.135 \\
\hline $\mathrm{Si} 3$ & 0.3904 & 0.0 & 0.1607 & 0.435 & 0.0 & 0.160 \\
\hline $\mathrm{Si} 4$ & 0.6667 & 0.3333 & 0.2108 & 0.667 & 0.333 & 0.210 \\
\hline Si5 & 0.6667 & 0.3333 & 0.3404 & 0.667 & 0.333 & 0.340 \\
\hline Si6 & 0.3895 & 0.0 & 0.2872 & 0.390 & 0.0 & 0.290 \\
\hline $\mathrm{Si} 7$ & 0.4215 & 0.2108 & 0.3470 & 0.474 & 0.237 & 0.345 \\
\hline $\mathrm{Si} 8$ & 0.2544 & 0.1272 & 0.4407 & 0.254 & 0.127 & 0.440 \\
\hline $\mathrm{O} 1$ & 0.6667 & 0.3333 & 0.0 & 0.667 & 0.333 & 0.0 \\
\hline $\mathrm{O} 2$ & 0.5411 & 0.2705 & 0.0822 & 0.625 & 0.250 & 0.120 \\
\hline $\mathrm{O} 3$ & 0.3942 & 0.1048 & 0.1348 & 0.440 & 0.122 & 0.125 \\
\hline $\mathrm{O} 4$ & 0.5433 & 0.2726 & 0.1882 & 0.540 & 0.270 & 0.180 \\
\hline O5 & 0.6667 & 0.3333 & 0.2755 & 0.667 & 0.333 & 0.240 \\
\hline O6 & 0.3763 & 0.0 & 0.2239 & 0.405 & 0.0 & 0.215 \\
\hline $\mathrm{O} 7$ & 0.5 & 0.0 & 0.1449 & 0.5 & 0.0 & 0.115 \\
\hline O8 & 0.5 & 0.0 & 0.3021 & 0.5 & 0.0 & 0.305 \\
\hline O9 & 0.3945 & 0.1063 & 0.3116 & 0.409 & 0.112 & 0.305 \\
\hline O10 & 0.5469 & 0.2735 & 0.3638 & 0.540 & 0.270 & 0.320 \\
\hline O11 & 0.3536 & 0.1768 & 0.4014 & 0.405 & 0.202 & 0.400 \\
\hline $\mathrm{O} 12$ & 0.1835 & 0.0 & 0.4300 & 0.244 & 0.0 & 0.425 \\
\hline $\mathrm{O} 13$ & 0.3017 & 0.1508 & 0.5 & 0.311 & 0.156 & 0.5 \\
\hline
\end{tabular}

reflection row revealed $a=14.51 \pm 0.10 \AA$ and, from the plot of the three-dimensional reciprocal lattice, $c=25.5 \pm 0.7 \AA$. Another MCM-49 sample gave a value $c=26.1 \pm 0.9 \AA$. These values can be compared to X-ray results: $a=14.28, c=25.37 \AA$ (Lawton et al., 1996). For this particular tilt series, $h k 0, h, k, 2 k$, $h, 2 n, 3 n, h k k, h, 4 n, 3 n, h, 3 n, 2 n$ and $h, 2 n, n$ sets were merged into a set of 137 unique reflections. The maximum tilt value used was $48.5^{\circ}$. During the merging process, presumed equivalent reflections in these sets were found experimentally to have intensity values near one another, again justifying the choice of the $P 6 / \mathrm{mmm}$ space group.

An attempt was made to expand a reasonable $h k 0$ phase set into three dimensions with the Sayre equation via a strong $h k u$ origin-defining reflection but this was not successful. [Only one $h k u$ reflection, where $u$ denotes an odd $l$ index, can be chosen for this space group (Rogers, 1980).] Clearly, index connectivity into the $3 \mathrm{D}$ set was poor. Although the selection and permutation of several algebraic unknowns might have been more effective, it was decided to change the approach to the $3 \mathrm{D}$ phasing problem and to use maximum entropy and likelihood.

An initial 3D phase determination based on maximum entropy and likelihood was carried out with the HadamardHamming sequence for eight phase generators. A useful $h k 0$ map was obtained (Fig. 5a) corresponding to a r.m.s. phase error of $50^{\circ}$ for the zone. The three-dimensional determination was not useful, i.e. $\langle\Delta \phi\rangle=83.7^{\circ}$. (A subset of 86 reflections had been assigned phase values.) Using a NordströmRobinson permutation sequence for 16 generators, MICE found a better 3D solution (Fig. $5 b$ ), apparent after the second origin choice ( $c / 2$ shift) was tested with the ITQ-1 framework model. In this case, $\langle\Delta \phi\rangle=46.7^{\circ}$ for the $h k 0$ data and $58.6^{\circ}$ for the complete $h \mathrm{kl}$ set. (Of the subset of 129 reflections assigned 
phase values, there are 47 erroneous estimates.) The phase shift is justified since the 233 origin-defining reflection selected by the MICE program had been assigned the default phase value 0 when the structural model in the other origin choice specifies a value $\pi$. It is clear that the observed density serves as a suitable envelope function for the framework $T$-site positions (Fig. 6). The most accurate phase set was found at the fourth largest maximum-likelihood value in the ranked list of 256 trial solutions.

If it is accepted that the ITQ-1 framework model (Fig. 1, Table 2) can account for the diffraction data from MCM-49, a structure-factor calculation for first-cited material gives $R(I)=$ $0.33, R(F)=0.36$. It is apparent that multiple-scattering effects lead to artificially high values for weak reflections since, for one sample, a subset of $33|F \mathrm{obs}| \geq 10, R(I)=R(F)=0.23$. For another independently synthesized sample, a subset of 23 largest reflections gives the same $R$ values as the model. In a test of the $3 \mathrm{D}$ data set from the second-cited sample, the FOMs from a structure-factor calculation for a set of 102 unique reflections are quite similar.

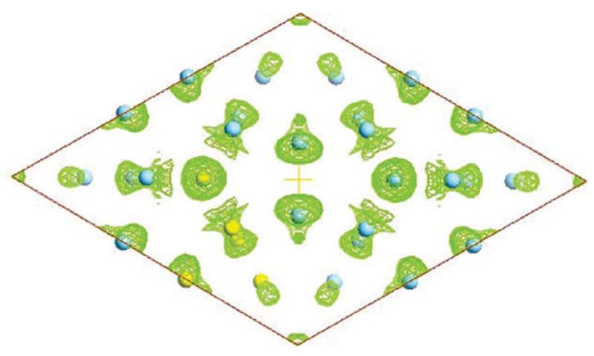

(a)

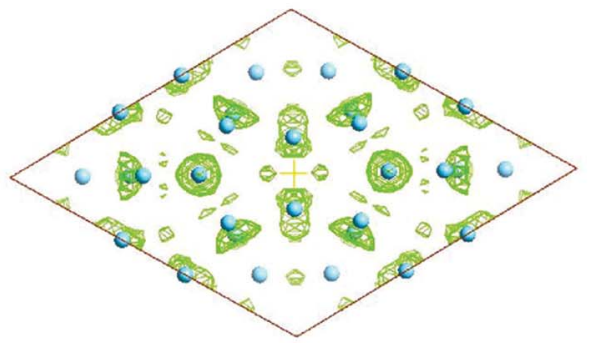

(b)

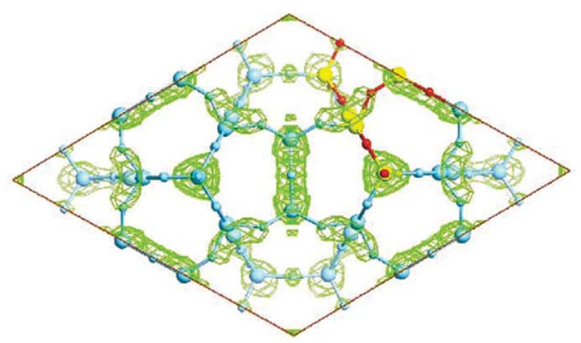

(c)

Figure 5

Interpretable [001] maps after maximum-entropy and likelihood determinations. (a) MCM-49 (Hadamard-Hamming code); (b) MCM-49 (Nordström-Robinson code); (c) composite MWW (NordströmRobinson code). Yellow spheres represent $T$ sites in the asymmetric unit. In $(c)$, all atomic positions are indicated.

\section{3. $\mathrm{MCM}-22$}

A zonal phase determination had been reported previously (Dorset, 2003d), given an electron diffraction pattern with p6mm symmetry. Tilt experiments around $a^{*}$ to assemble a 3D set of 104 unique reflections finds unit-cell values: $a=$ $14.43 \pm 0.09, c=24.8 \pm 0.8 \AA$. This comprises $h k 0, h k k$ and $h, k, 2 k$ zonal sets within a maximum tilt of $49.5^{\circ}$. The previous electron diffraction investigation had found values of $a=$ 14.30, $c=26.40 \AA$ whereas X-ray values have been reported (Leonowicz et al., 1994; Lawton et al., 1996) as $a=14.11, c=$ 24.88 or $a=14.27, c=25.16 \AA$. Again the symmetry relationships among intensities are consistent with space group P6/mmm.

A structure-factor calculation based on the ITQ-1 model determines $R(I)=0.36, R(F)=0.40$ but, for a subset of 26 reflections with $\left|F_{\mathrm{obs}}\right| \geq 10, R(I)=R(F)=0.26$. Based on the structure-factor calculation, there is no $3 \mathrm{D}$ justification for the detrital atoms invoked by Leonowicz et al. (1994), just as there had been none for the determination based on $h k 0$ reflections

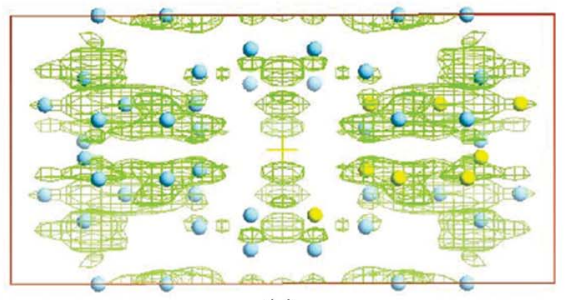

(a)

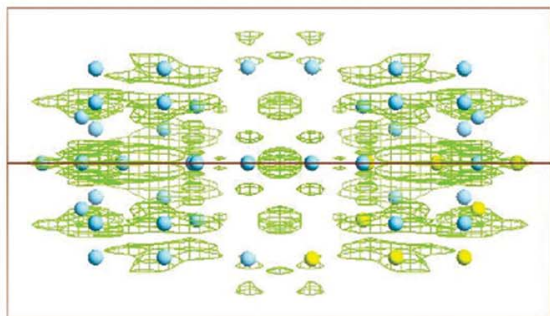

(b)

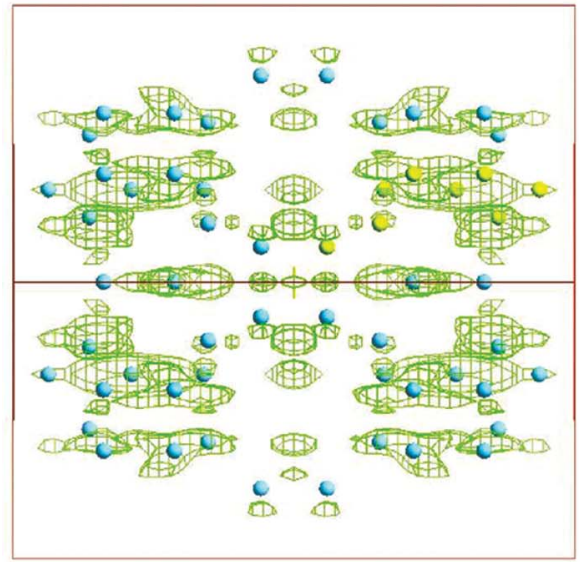

(c)

Figure 6

Nordström-Robinson phasing of MCM-49 - projections normal to the $c$ axis. (a) Projection down the $a$ axis; (b) [110] projection; (c) [110] projection. Spheres represent $T$-site positions. 
(Dorset, 2003d). An ab initio 3D phase determination was not attempted with this data set.

\subsection{ITQ-2}

From the three-dimensional tilt series on this material, it was clear that the reciprocal-lattice points along $\mathbf{c}^{*}$ are extensively streaked, denoting a lamellar stacking of only a few unit cells [i.e. 'shape-transform' broadening as the Fourier transform of a small number of unit cells (e.g. see Cowley, 1990; Dorset, 1995)]. It is not possible, therefore, to estimate a value for a $c$ axis from the reciprocal-lattice plots. The observed in-plane dimension, $a=14.36 \pm 0.09 \AA$, again agrees with previously cited measurements on similar materials (see above). Nevertheless, the microcrystalline plates are quite flat so that symmetric $h k 0$ patterns with $p 6 \mathrm{~mm}$ symmetry are often observed and are similar to Fig. 2(a).

It is legitimate to ask if the unit cell of the three-dimensional material remains intact within this structure. To investigate this, the continuously streaked $3 \mathrm{D}$ pattern can be sampled with an approximately $c=25 \AA$ unit-cell length to assemble a quasi-periodic $3 \mathrm{D}$ crystal data set comprising 85 unique reflections. This represents the selection of $h k 0, h k k$ and $h, k, 2 k$ zones within a maximum $55.5^{\circ}$ tilt around $a^{*}$. Comparison of the ITQ-1 framework model to these measured amplitudes via a structure-factor calculation yields $R(I)=0.34, R(F)=0.39$, consistent with the agreement found for true three-dimensional diffraction data. In terms of strong reflections, i.e. 25 reflections where $|F \mathrm{obs}| \geq 10, R(I)=R(F)=$ 0.23 .

\subsection{Composite MWW data set}

As mentioned above, the 3D intensity data from the three calcined zeolites with 3D frameworks are closely similar to one another. If the frameworks are closely similar, then the intensities must be very similar because the electron scattering factors (Doyle \& Turner, 1968) of Al and Si atoms in various aluminosilicate compositions are not easily distinguished. Thus, a composite three-dimensional intensity set of 155 unique reflections was built up from these separate data sets (viz MCM-22, MCM-49, ITQ-1), again in space group $P 6 / \mathrm{mmm}$, and termed MWW. Monitoring 78 recurrent $h k 0$, $h k k, \quad h, k, 2 k$ reflections, values of $R_{\text {merge }}=\sum|| F(X) \mid-$ $k|F(\mathrm{MWW})| / \sum|F(X)|=0.12$ when $X=\mathrm{MCM}-49,0.14$ when $X=$ ITQ-1 and 0.10 when $X=$ MCM-22. (A similar test of ITQ-2 data found $R_{\text {merge }}=0.13$.) The data extend to a diffraction limit $d_{\min }=1.33 \AA$. In previous studies of materials with the MWW framework structure, discussed above, probabilistic direct-methods phase determinations were somewhat successful for assigning values to $h k 0$ amplitudes in the view down the unit-cell hexagonal axis. However, symbolic addition was most effective for MCM-49 and ITQ-1 data whereas the only useful phase set for MCM-22 was found from a punctured Hadamard-Hamming set of seven strong reflections (Dorset, 2003d). Promising 3D results from MCM-49 encouraged the maximum-entropy and likelihood attempt on a larger composite 3D data set.
Although several error-correcting code options were explored, the best result was achieved when the NordströmRobinson basis set of 16 reflections was permuted and also assigning a value for $\varphi_{233}=\pi$, to set an origin identical to the one selected in the usual structural representation of this framework. From this starting set, 256 trial solutions were generated. For map evaluation, the top 30 solutions, ranked in order of decreasing LLG, were inspected. Care was taken to dampen the convergence of entropy maximization for each trial map so that $\chi^{2}$ would be acceptably close to 1.0. Several maps were found with considerable density near the unit-cell origin with no features in the rest of the cell, so these could be easily rejected. A few were identified with suitable solutions of the $h k 0$ phase set but with no fit to the model in the 3D density profile. Two solutions were found, one at the 20th position in the LLG list and the other at the 26th, where there was a reasonable match of density profile to the known structural model. In the first solution, also corresponding to the 7th most positive map in terms of entropy, the mean phase error for $h k 0$

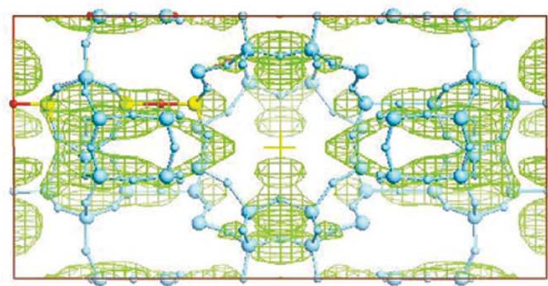

(a)

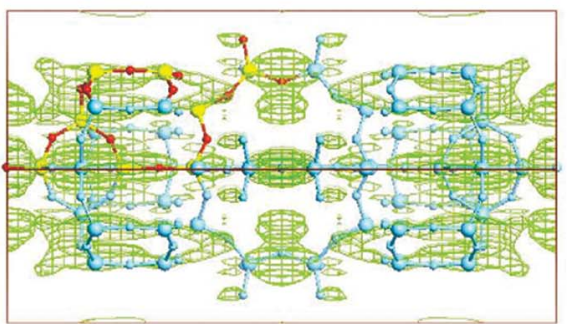

(b)

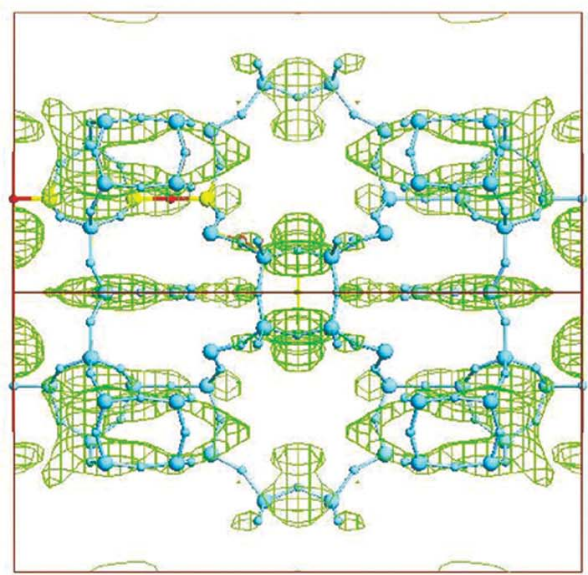

(c)

Figure 7

Nordström-Robinson phasing of composite MWW - projections normal to the $c$ axis. (a) Projection down the $a$ axis; (b) [110] projection; (c) [110] projection. All atomic sites are shown and yellow/red sections indicate the asymmetric unit of the framework. 
reflections was $63.0^{\circ}$ and for the 103 phased $h \mathrm{kl}$ reflections the error was $76.9^{\circ}$ or $60.0^{\circ}$ for the 27 strongest reflections. In the other solution, the respective phase errors were: $45.0,76.9$ and $53.3^{\circ}$.

Taking the solution with the best phase fit, one immediately sees (Fig. $5 c$ ) that the density profile accurately outlines the actual projected framework atom sites, including the O-atom positions in the view down [001]. In the views normal to the hexagonal axis, e.g. along the $a$ axis, as well as [110] and [110] projections (Fig. 7), the density profile provides mostly a good envelope for the framework structure but does not identify the positions of individual atoms.

An attempt was then made to improve the structure by Fourier refinement. It is possible to identify the $T$-site positions and many of the connecting $\mathrm{O}$ atoms. However, there is some ambiguity in the $z / c$ positions of these atoms. In the most optimistic case, it is possible to find a mean phase fit $\langle\Delta \varphi\rangle=$ $49.0^{\circ}$ for all 155 observed reflections. (This corresponds to 39 erroneous phases in the whole reflection set, given in Table 1.) However, owing to unobserved data within the missing cone, it is not possible to locate the atomic positions accurately enough in the $c$ direction to construct a model directly from the potential map slices so that it can be optimized by a DLS (distance least squares) refinement (Baerlocher et al., 1977). However, from a geometrical fit, with known bond distances and angles, it might be possible to construct such a model if the $\mathrm{Si}-\mathrm{O}$ framework is constructed around the density profile.

\section{Discussion}

\subsection{Comparison of MWW materials}

By contrast to experience with organic materials (Dorset, 1995), the electron crystallography of silicates presents new challenges. Simply stated, the selected-area electron diffraction intensities are less satisfactory for direct structure analysis. For many organics, realistic crystal structure solutions can be found from electron diffraction intensities resulting in $R(F) \leq 0.25$ (Dorset, 1995), perhaps corresponding to results from the early days of X-ray crystallography. In fact, data from the MWW family of materials represent an optimal case compared to data sets from other silicate materials (e.g. Dorset, 2003b,c). Multiple-scattering effects are observed even when perfect crystallite thicknesses [e.g. indicated from diffraction resolution and a simple shape transform argument (Dorset, 2003b)] would presuppose intensities closer to a single-scattering model. Indeed, these multiple-scattering perturbations seem to be insensitive to the accelerating voltage used for data collection, something not expected for coherent dynamical scattering (Cowley, 1990). For example, intensity data from ZSM-5 taken at 100, 200 and $300 \mathrm{kV}$ cannot be easily distinguished with an $R$-value comparison to the structure model.

With these caveats in mind, it is clear, nevertheless, that all of the materials examined in this study are closely related to one another. Again, all of the raw 3D data sets from comparable materials strongly resemble one another. As revealed by $a b$ initio structure analysis, the three-dimensional structures of calcined MCM-22, MCM-49 and ITQ-1 share the same MWW framework, perhaps representing different combinations of $\mathrm{Al}$ and $\mathrm{Si}$, but packing in the hexagonal space group $P 6 / \mathrm{mmm}$. There is no case to be made for $\mathrm{Cmmm}$ as a viable space group for these materials. There also seems to be no justification for 'detrital' atoms in addition to the framework. Nor is there any evidence for large channels parallel to the [001] direction for some materials.

The previous rationale for postulating the $\mathrm{Cmmm}$ framework (Leonowicz et al., 1994) was invoked to avoid a $180^{\circ}$ $T_{1}-\mathrm{O}_{1}-T_{1}$ bond angle connecting $\left\{4^{3} 5^{6} 6^{3}\left[4^{3}\right]\right\}$ cages. This is based on a common assumption that such linkages truly represent an average over various bond angles (Lawton \& Rohrbaugh, 1990). This assumption, however, may be false. For example, theoretical phenomenological force-field calculations (Pilati et al., 1998) that fit vibrational frequencies in several silicates accurately predict atom displacement parameters in the mineral coesite, as well as other thermodynamic parameters. The calculation also justifies a linear $\mathrm{Si}-\mathrm{O}-\mathrm{Si}$ angle in this material. Other covalent potential models of two silicates, including coesite, show that the barrier to the linearity of this linkage at an equilibrium bond distance value is only $3 \%$ of the $\mathrm{Si}-\mathrm{O}$ bond energy (Stixrude \& Bukowinski, 1988). In other words, packing forces may predispose such linear Si-atom linkages through $\mathrm{O}$ atoms. Compressibility studies reveal that intra-tetrahedral forces are very strong compared to inter-tetrahedral ones, so that the linkages, e.g. $\mathrm{Si}-\mathrm{O}-\mathrm{Si}$, are most easily deformed. In other words, the linear bond angle in MWW may actually be present and not merely represent an averaging effect of the hexagonal space group. Even in two dimensions, the hexagonal vs orthorhombic symmetry can be established by calculation of $h k 0$ intensity data. The $\mathrm{cmm}$ projection of $\mathrm{Cmmm}$ contains extra reflections that would violate the observed $p 6 \mathrm{~mm}$ symmetry. However, $p 6 \mathrm{~mm}$ is clearly indicated in all $h k 0$ electron diffraction patterns from these materials (e.g. Fig. 2).

Although a subtle difference was proposed earlier for MCM-22 and MCM-49, based on zonal electron diffraction data (Dorset, 2003d), it is now clear that the slight changes in the diffraction intensity distributions must arise from a source other than a structural one. It might, for example, represent a difference of average crystal thicknesses. For example, two groups of $h k 0$ patterns were also noted for ITQ-1 (see above), where one intensity distribution obviously (by visual inspection) arose from thinner crystals. Given the crystal structure, the other intensity distribution could be accounted for by a secondary scattering calculation, assuming a greater average crystal thickness for the second pattern. From the fit of the structural model to the observed intensities, it follows that MCM-22 would form thinner crystals than would MCM- 49 , in accord with the different synthetic paths (Rubin \& Chu, 1990; Bennett et al., 1993). A test of this hypothesis, via a secondary scattering calculation (Cowley et al., 1951), reveals that the correction to $\left|F_{\text {calc }}\right|$ via $I^{\prime}=I_{\text {calc }}+I_{\text {calc }} \otimes I_{\text {calc }}$ (where $\otimes$ denotes convolution) improves the agreement of the observed $h k 0$ intensities for MCM-49 by lowering $R(F)$ from 0.40 to e.g. 0.25 . 
Hence the criterion of data self-consistency as a test of 'sound' intensity data should be used with caution in the electron crystallography of inorganic materials, especially if there is a narrow distribution of crystal thicknesses for a particular material.

The product of a delaminated MCM-22 precursor, the ITQ2 sample examined in this work, appears to be a closely related material to the others, if only for the average number of unit cells found in crystalline lamellar stacks. Evidenced by the streaking of reflections along $\mathbf{c}^{*}$, there are only a small number of unit cells in the crystal stack in ITQ-2. Contradicting the postulate of a disrupted surface structure of such materials, caused by exfoliation (Corma et al., 2000), it is found that the total atomic framework of the ITQ-1 model accounts for the intensity data of the electron diffraction pattern. Indeed, occasional MCM-22 samples comprising one or two unit-cell layers have been observed (Lawton et al., 1998).

\subsection{Direct phase determination - success and limitations}

From the results presented above, it is also clear that the maximum-entropy and likelihood approach, using errorcorrecting codes for phase permutation, can be quite successful for assigning phase values to incomplete threedimensional electron diffraction amplitudes from goniometric tilting experiments. The difficulties encountered in this procedure for this direct determination of the MWW zeolite crystal structure are purely a matter of the incomplete data sampling, i.e. due to the missing cone of diffraction information incurred by a restricted goniometer tilt range (Dorset \& McCourt, 1993). This is demonstrated by the elongation of density along the $c$-axis direction in Figs. 6 and 7. Nevertheless, since the crystal structure of MCM-22, a member of the MWW family, was originally elucidated solely from high-resolution images of its structure in two orthogonal projections (Leonowicz et al., 1994), the higher resolution provided by the current electron diffraction determination in three dimensions should provide a superior constraint to the construction of a three-dimensional model.

One of the interesting aspects of this determination is that some additional effort may be required to arrive at a more robust FOM for ready identification of the best structural solution in the list of phase sets ranked according to LLG. With X-ray data, it is usual to find the best solution, in terms of phase errors, in the top eight phase sets ranged by LLG. This is not the case for electron diffraction data. It is clear that many trial solutions can be rejected out of hand immediately from the appearance of the maximum-entropy maps because the density distributions do not correspond to any rational structural detail. On the other hand, at higher values of LLG for the composite MWW data, there are at least three acceptable phase sets for $h k 0$ data that are worthless when considered in three dimensions and these are found before the first solution with reasonable three-dimensional density features. The problem arises because the LLG uses all of the observed data, not just the strong reflections, and the weak reflections are subject to error because of multiple scattering.
It is also assumed that the reflections are independent but it is clear, from the Sayre equation trials, that this is not the case. It may be that the use of a non-diagonal approximation to the LLG estimate could be of value as an improved FOM. How the decision of the best possible map might be made for a completely unknown structure, therefore, is open to question. Two reasonable solutions were found in the top 30 phase sets in a complete sampling of 256 trials for the composite data set and at the 4th largest LLG value for MCM-49, so these favorable solutions are also not randomly distributed.

It may be that these concerns will disappear when complete three-dimensional electron diffraction intensities are available, since the structural uncertainty due to the missing cone of reflections prohibits identification of the framework in terms of atomic positions but multiple scattering effects would, of course, still be present. (Again, absence of the $00 l$ intensity information is just as detrimental for this determination as would be the loss of diffraction peaks along a fiber axis for a polymer structure determination.) How threedimensional data are to be completed is yet unsolved. Earlier work, e.g. on SSZ-48 (Wagner et al., 1999) had suggested the use of crystals cleaved in an ultramicrotome. However, it is unusual to obtain sections less than $500 \AA$ thick and, because the materials are brittle, they tend to shatter or suffer mechanical damage during this operation. In other words, the intensities in the missing cone region recorded from such samples may be less than optimal. It might be found that crystals sectioned with a focused ion-beam source (Overwijk $e t$ al., 1993) will yield improved electron diffraction intensities. Shattering is of no concern and the section thicknesses should be less than those obtained with an ultramicrotome. Also the layer of amorphous material left after such sectioning experiments should be of little concern to electron diffraction experiments.

Absent amplitude and phase terms might also be predicted by extending the existing information into the missing cone region, e.g. by the Sayre (1952) equation or maximum-entropy extrapolation. The former strategy has been exploited previously for a polysaccharide (see Dorset \& McCourt, 1993, 1999). For the composite MWW set, a preliminary trial of phase and amplitude expansion was made from the Fourierrefined phase set (Table 1) into a set of 117 unknowns. The phase error $\langle\Delta \varphi\rangle$ was found to be $78.5^{\circ}$ for the whole set, $67.9^{\circ}$ for the 62 most intense reflections and $55.4^{\circ}$ for the $260 \mathrm{kl}$ reflections. (A random determination has an error of $90^{\circ}$.) The prediction of $\mid F$ obs $\mid$ was not particularly good. Clearly, addition of some valid information to the 'missing cone' region will begin to localize atomic positions along the $c$ axis in resulting potential maps. This is most successful for finding $T$-site positions where the mean positional error was $0.16 \AA$. The $\mathrm{O}$-atom positions are more difficult to locate and the mean positional error (Table 2) was $0.50 \AA$. (Admittedly also, the known structural model was used to search for atomic positions.) A structural model constructed from found $T$-site positions, with theoretical O-atom connections located in the 3D maps and/or established from approximate theoretical positions between $\mathrm{Si}$ atoms, is depicted in Fig. 8, compared to 
the actual ITQ-1 model in Fig. 1. Although the bonding is distorted, particularly in the $4^{3} 5^{5} 6^{3}\left[4^{3}\right]$ cage, it may be possible to construct a valid framework model from this phasing result.

\section{Future prospects}

It should be emphasized here that the layered zeolite examined in this study presents a somewhat optimized case of a material with minimal crystal thickness so that secondary scattering perturbations to the intensity data are not too severe. Also, for most zeolites, interesting structural details, such as pore openings etc., are not commonly observed in the projection onto the major crystal face. A significant challenge to data collection will be presented by more 'typical' threedimensional zeolites where the crystals may be somewhat thicker and the most available projection is one onto the most densely packed crystal face. Incomplete crystal tilts would then frustrate the characterization of pore channel geometries since diffraction information about the pores would lie in a least expressed crystal face (i.e. not a commonly expressed zonal projection) and/or outside the range of the goniometric tilt range. This provides further incentive to develop techniques for sectioning crystals to orient the structurally most informative projections into the electron-beam path, as suggested above. To cope with increased crystal thickness,
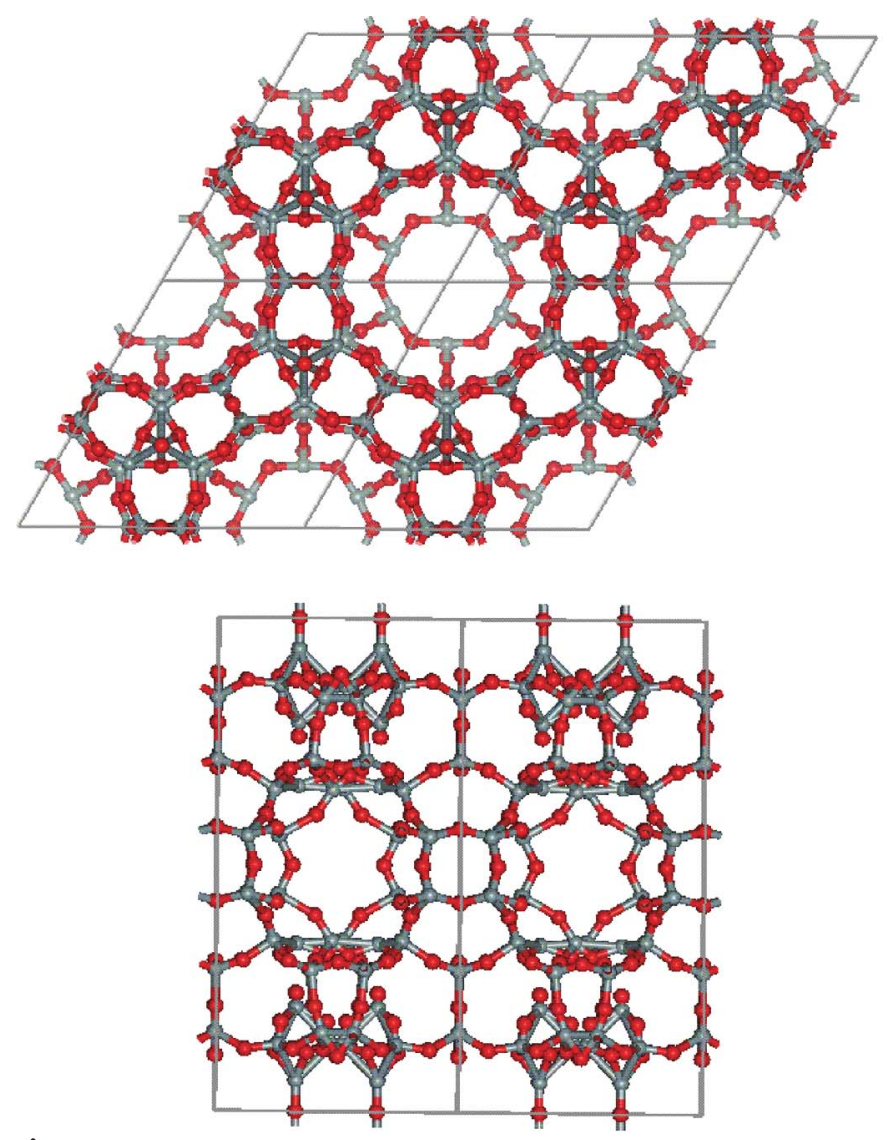

Figure 8

Model for MWW extracted from three-dimensional maps after Fourier refinement. Top: [001] projection, bottom: projection down the $a$ axis. tests are under way to evaluate the precession method for data collection to ascertain whether true integrated intensities will be useful for $a b$ initio structural determination, since the precession mode (Vincent \& Midgley, 1994) will presumably reduce the influence of multiple-scattering effects. [A preliminary test on ITQ-1 using a NanoMegas SpinningStar P010 attachment to the Philips CM-30 microscope indicates that the fit of the structural model to all $h k 0$ zonal precession data is improved, i.e. $R(I, F)=0.25$ and, moreover, that many of the spuriously intense reflections disappear when the pattern is built up via hollow-cone illumination.]

We acknowledge help from Wei Dong with the MICE computer program. Karl G. Strohmaier and Gordon J. Kennedy are thanked for a critical reading of the manuscript.

\section{References}

Baerlocher, Ch., Hepp, A. \& Meier, W. M. (1977). Distance Least Squares Refinement Program, DLS-76. ETH, Zürich.

Baerlocher, Ch., Meier, W. M. \& Olson, D. H. (2001). Atlas of Zeolite Framework Types, 5th, revised ed., pp. 202-203. Amsterdam: Elsevier.

Bennett, J. M., Chang, C. D., Lawton, S. L., Leonowicz, M. E., Lissy, D. N. \& Rubin, M. K. (1993). US Patent 5,236,575.

Bricogne, G. \& Gilmore, C. J. (1990). Acta Cryst. A46, 284-297.

Camblor, M. A., Corma, A., Diaz-Cabanas, M. J. \& Baerlocher, Ch. (1998). J. Phys. Chem. B, 102, 44-51.

Chan, I. Y., Labun, P. A., Pan, M. \& Zones, S. I. (1995). Micropor. Mater. 3, 409-418.

Cheetham, A. K. (1995). The Rietveld Method, edited by R. A. Young, pp. 276-292. Oxford University Press.

Chu, C. T. W., Kresge, C. T., Roth, W. J., Simmons, K. G. \& Vartuli, J. C. (1994). US Patent 5,292,698.

Corma, A., Formes, V., Guil, J. M., Pergher, S. B., Maesen, Th. L. M. \& Buglass, J. G. (2000). Micropor. Mesopor. Mater. 38, 301-309.

Corma, A., Formes, V., Pergher, S. B., Maesen, Th. L. M. \& Buglass, J. G. (1998). Nature (London), 396, 353-356.

Cowley, J. M. (1990). Diffraction Physics, 2nd ed. Amsterdam: NorthHolland.

Cowley, J. M. \& Moodie, A. F. (1962). J. Phys. Soc. Jpn, 17, Suppl. B-II, 86-91.

Cowley, J. M., Rees, A. L. G. \& Spink, J. A. (1951). Proc. Phys. Soc. London Sect. A, 64, 609-619.

Diaz, I., Kokkoli, E., Terasaki, O. \& Tsapatsis, M. (2004). Chem. Mater. 16, 5226-5232.

Diaz-Cabanas, M. J., Camblor-Fernandez, M. A., Corell-Martires, C. \& Corma-Canos, A. (2000). US Patent 6,077,498.

Dorset, D. L. (1995). Structural Electron Crystallography. New York: Plenum.

Dorset, D. L. (2003a). Z. Kristallogr. 218, 237-246.

Dorset, D. L. (2003b). Z. Kristallogr. 218, 458-465.

Dorset, D. L. (2003c). Z. Kristallogr. 218, 525-530.

Dorset, D. L. (2003d). Z. Kristallogr. 218, 612-616.

Dorset, D. L. \& McCourt, M. P. (1993). J. Struct. Biol. 111, 118-124.

Dorset, D. L. \& McCourt, M. P. (1999). Adv. X-ray Anal. 41, 614-628.

Doyle, P. A. \& Turner, P. S. (1968). Acta Cryst. A24, 390-397.

Fung, A. S., Lawton, S. L. \& Roth, W. J. (1994). US Patent 5,362,697.

Gilmore, C. J. (1998). Direct Methods for Solving Macromolecular Structures, edited by S. Fortier, pp. 159-167. Dordrecht: Kluwer Academic Publishers.

Gilmore, C. J. \& Bricogne, G. (1997). Methods Enzymol. 277, 65-78. Gilmore, C. J., Bricogne, G. \& Bannister, C. (1990). Acta Cryst. A46, 297-308. 
Gilmore, C. J., Dong, W. \& Bricogne, G. (1999). Acta Cryst. A55, 70-83.

Golay, M. J. E. (1949). Proc. IEEE, 37, 657.

Hahn, Th. (1995). International Tables for Crystallography, Vol. A, Space Group Symmetry. Dordrecht: Kluwer Academic Publishers.

Hauptman, H. A. (1972). Crystal Structure Determination. The Role of the Cosine Seminvariants. New York: Plenum.

Karle, J. \& Hauptman, H. (1956). Acta Cryst. 9, 635-651.

Karle, J. \& Karle, I. L. (1966). Acta Cryst. 21, 849-859.

Lawton, S. L., Fung, A. S., Kennedy, G. J., Alemanny, L. B., Chang, C. D., Hatzikos, G. H., Lissy, D. N., Rubin, M. K., Timken, H. K. C., Steuernagel, S. \& Woessner, D. E. (1996). J. Phys. Chem. 100, 3788-3798.

Lawton, S. L., Leonowicz, M. E., Partridge, R. D., Chu, P. \& Rubin, M. K. (1998). Micropor. Mesopor. Mater. 23, 109-117.

Lawton, S. L. \& Rohrbaugh, W. J. (1990). Science, 247, 1319-1322.

Leonowicz, M. E., Lawton, J. A., Lawton, S. L. \& Rubin, M. K. (1994). Science, 264, 1910-1913.

Luzzati, V., Mariani, P. \& Delacroix, H. (1988). Makromol. Chem. Macromol. Symp. 15, 1-17.

McCusker, L. B. (1991). Acta Cryst. A47, 297-313.

Morris, R. E., Burton, A., Bull, L. M. \& Zones, S. I. (2004). Chem. Mater. 16, 2844-2851.

Nicolopoulos, S., Gonzalez-Calbet, J. M., Vallet-Regi, M., Camblor, M. A., Corell, C., Corma, A. \& Diaz-Cabanas, M. J. (1997). J. Am. Chem. Soc. 119, 11000-11005.

Nicolopoulos, S., Gonzalez-Calbet, J. M., Vallet-Regi, M., Corma, A., Corell, C., Guil, J. M. \& Perez-Pariente, J. (1995). J. Am. Chem. Soc. 117, 8947-8956.

Njo, S. L., Van Koningsveld, H., Van de Graaf, B., Baerlocher, Ch. \& McCusker. L. B. (1999). Proceedings of the 12th International Zeolite Conference, pp. 2519-2524, Warrendale, PA: Materials Research Society.

Nordström, A. W. \& Robinson, J. P. (1967). Inform. Contrib. 11, 613-616.
Overwijk, M. N. F., van den Heuvel, F. C. \& Bulle-Lieuwma, C. W. T. (1993). J. Vac. Sci. Technol. B11, 2021-2024.

Pilati, T., DeMartin, F. \& Gramaccioli, C. M. (1998). Phys. Chem. Miner. 25, 152-159.

Puppe, L. \& Weisser, J. (1984). US Patent 4,439,409.

Robertson, J. M. \& White, J. G. (1945). J. Chem. Soc. pp. 607-617.

Rogers, D. (1980). Theory and Practice of Direct Methods in Crystallography, edited by M. F. C. Ladd \& R. A. Palmer, pp. 23-92. New York: Plenum.

Roth, W. J., Kresge, C. T., Vartuli, J. C., Leonowicz, M. E., Fung, A. S. \& McCullen, S. B. (1995). Stud. Surf. Sci. Catal. 94, 301-307.

Roth, W. J. \& Vartuli, J. C. (2002). Stud. Surf. Sci. Catal. 141, 273-280.

Rubin, M. K. \& Chu, P. (1990). US Patent 4,954,325.

Sato, T. (1992). Acta Cryst. A48, 842-850.

Sayre, D. (1952). Acta Cryst. 5, 60-65.

Sayre, D. (1993). Acta Cryst. D49, 107.

Stixrude, L. \& Bukowinski, M. S. T. (1988). Phys. Chem. Miner. 16, 199-206.

Szostak, R. (1998). Molecular Sieves. Principles of Synthesis and Identification, $2 \mathrm{~d}$ ed. London: Blackie Academic \& Professional.

Terasaki, O. \& Ohsuna, O. (2003). Topics Catal. 24, 13-18.

Thomas, J. M., Terasaki, O., Gai, P. L., Zhou, W. \& Gonzalez-Calbet, J. (2001). Acc. Chem. Res. pp. 583-594.

Vainshtein, B. K. (1964). Structure Analysis by Electron Diffraction. Oxford: Pergamon.

Vincent, R. \& Midgley, P. A. (1994). Ultramicroscopy, 53, 271-282.

Wagner, P., Terasaki, O., Ritsch, S., Nery, J. G., Zones, S. I., Davis, M. E. \& Hiraga, K. (1999). J. Phys. Chem. B, 103, 8245-8250.

Weirich, T. E. (2001). Acta Cryst. A57, 183-191.

Wilson, A. J. C. (1942). Nature (London), 150, 151-152.

Woolfson, M. M. (1954). Acta Cryst. 7, 65-67.

Zachariasen, W. H. (1952). Acta Cryst. 5, 68-73.

Zones, S. I., Holtermann, D. I., Innes, R. A., Pecaoro, T. A., Santilli, D. S. \& Ziemer, J. N. (1989). US Patent 4,826,667.

Zou, X. D., Sukharev, Yu. \& Hovmöller, S. (1993). Ultramicroscopy, 49, 147-158. 